\title{
Virtual Screening of Inhibitors for Chitosanases EAG1
}

\author{
Xiangyang Xu*, Zaiwei Song, Yunchao Yin, Faguo Zhong, Junying Song, Jiachao Huang, \\ Wangli Ye, Peng Wang
}

Zaozhuang Jienuo Enzyme Co., Ltd., Zaozhuang City, China

Email: ${ }^{\star}$ sdjienuo@163.com

How to cite this paper: $\mathrm{Xu}, \mathrm{X} . Y .$, Song, Z.W., Yin, Y.C., Zhong, F.G., Song, J.Y., Huang, J.C., Ye, W.L. and Wang, P. (2020) Virtual Screening of Inhibitors for Chitosanases EAG1. Advances in Enzyme Research, 8, 49-57.

https://doi.org/10.4236/aer.2020.84005

Received: December 15, 2020

Accepted: December 27, 2020

Published: December 30, 2020

Copyright $\odot 2020$ by author(s) and Scientific Research Publishing Inc. This work is licensed under the Creative Commons Attribution International License (CC BY 4.0).

http://creativecommons.org/licenses/by/4.0/

\begin{abstract}
Chitosanases EAG1 is a classical glycoside hydrolase from Bacillus ehimensis. The previous researches showed that this Chitosanases can not only hydrolyze the b1,4-glycosidic bonds of chitosan to COS in different sizes but also keep a high catalytic activity in organic, which was useful for producing chitooligosaccharides and GlcN for use in the food and pharmacological industries. While it is instable in the liquid state. This shortcoming seriously restricts its industrial application. Here we used the modeled structure of EAG1 and the molecular modeling software package to screen the free chemical database ZINC. Moreover, the strategies including "initial filter" and consensus scoring were applied to accelerate the process and improve the success rate of virtual screening. Finally, five compounds were screened and they were purchased or synthetized to test their binding affinity against EAG1. The test results showed that one of them could inhibit the enzyme with an apparent $K_{\mathrm{i}}$ of $1.5 \mu \mathrm{M}$. The result may take the foundation for further inhibitor screening and design against EAG1 and the screened compound may also help to improve the liquid stability of EAG1 and expand its industrial application.
\end{abstract}

\section{Keywords}

Chitosanases, Inhibitor, Virtual Screening

\section{Introduction}

Chitosanases belong to glycoside hydrolase families 5, 7, 8, 46, 75 and 80 and hydrolyse glucosamine polymers are produced by partial or full deacetylation of chitin [1]. They are of important industrial application in the utilization of the enormous chitosan and chitin substrates, available from sea-food-processing units, for the generation of the size-specific chitosan oligomers required particu- 
larly in pharmaceutical industries [2]. In industrial enzyme applications, stability is a key factor. In order to improve the stability of enzymes, a number of protein engineering techniques, such as random mutagenesis, DNA shuffling, truncation and circularization, have been developed for improving the stability of proteins [2]. However, there was not any work focusing on improving liquid stability for Chitosanases by adding inhibitors. This technology had been used on protease. The prior art has dealt extensively with improving the storage stability, for example, by adding a protease inhibitor [3] [4] [5]. The application of high concentration liquid enzyme preparation in industry will become the development direction in the future.

VS is a technique to identify novel hits (i.e., bioactive molecules) from large chemical libraries through computational means by applying knowledge about the protein target (structure-based VS) or known bioactive ligands (ligand-based VS). The ligand-based approaches utilize structure-activity data from a set of known actives in order to identify drug candidates for experimental evaluation. In the previous study, researchers have used virtual screening method to screen inhibitors of protease MP, and the obtained inhibitors have significantly improved the stability of the enzyme preparation [6]. EAG1 is a classical glycoside hydrolase from Bacillus ehimensis, it exhibits maximum activity at $50^{\circ} \mathrm{C}$. Not only can it hydrolyze the b1,4-glycosidic bonds of chitosan to COS in different sizes but also keeps a high catalytic activity in organic solvents with metal ions [7]. In the previous researches, its tertiary has been modelled [8] and a disulfide bridge in flexible regions can improve EAG1's thermo-stability and catalytic efficiency [7]. However, it is unstable in liquid state, which is the common disadvantage as all enzymes.

In the present study, we focused on database screening, biological evaluation and mechanism illumination of inhibitors for Chitosanases EAG1. Our virtual screening approach was based on initial high through-put docking calculations performed on a library of about $23,000,000$ commercially available compounds in the database of ZINC [9] [10]. The recompile software of Autodock4.2 was used for docking produce, consensus score, as well as the special filter strategy built based on the experimental and docking results of known inhibitors for Chitosanases were applied to improve the success rate of inhibitors finding. Three compounds were synthesized and assayed in vitro. One of them could inhibit EAG1 with apparent $K \mathrm{i}$ with $1.5 \mathrm{mM}$, which could be used for further inhibition mechanism detection. This finding provided the bases for further selection and synthesis of more effective inhibitors for Chitosanases EAG1.

\section{Material and Methods}

\section{Docking studies and the "initial filter" building}

The structure of EAG1 [7] was modeled in the previous work and it was clarified that all of the residues were in the acceptive zone. The modeled structure was defined as the acceptor. The active site was defined using AutoGrid and the 
center $(37.286,4.2688,25.31)$ of the box was chosen according to the position of key residue in pocket. The grid size was set to $60 \times 60 \times 60$ points with grid spacing of $10 \AA$ (Figure 1). The grid box included the entire binding site of the enzyme and the residues in active site were set flexible in the whole docking process. The small molecules were defined as ligands and the small molecular was set as flexibility ligand. That is to say, all rotational bonds were set free.

The Autodock4.2 program has been proved to be a robust approach with good docking accuracy and reliability in the docking of inhibitors [11] [12]. And the Autodock Tools (ADT) [13] was used for preparing molecules and all of the hydrogen was added by using REDUCE [14]. All of the docking decoys were clustered with cutoff 2 according to root mean square deviation (RMSD).

Previous studies of inhibitors for Chitosanases showed that some compounds had good capacities as Chitosanases inhibitors (Table 1). Hence, we used these compounds to train the characters and built an "initial filter" for the virtual screening. All of the character setting was just as the steps mentioned above. The tertiary structures of these compounds were listed in Figure 2. These structures were downloaded from Chemspider [15] (http://www.chemspider.com/).

Table 1. The experimental $K_{i}$ and docking $K_{i} E_{i}$ values for compounds in training set.

\begin{tabular}{|c|c|c|c|}
\hline Compounds & $K_{i}(\mu \mathrm{M})$ & Docking $K_{i}(\mu \mathrm{M})$ & Docking $E_{i}(\mathrm{kcal} / \mathrm{mol})$ \\
\hline Benzalkonium chloride & 2.36 & 8.89 & -4.16 \\
\hline 2-Hydroxy-5-nitrobenzyl bromide & 0.42 & 2.85 & -8.14 \\
\hline N-bromosuccinimide & 0.07 & 0.93 & -15.41 \\
\hline Sodium acetate & 0.48 & 2.29 & -8.62 \\
\hline p-chloromercuribenzoate & 0.20 & 1.61 & -9.54 \\
\hline p-hydroxymercuribenzoic acid & 0.16 & 1.46 & -10.69 \\
\hline 2-Mercaptoethanol & 0.02 & 0.49 & -12.64 \\
\hline Diethyl pyrocarbonate & 0.03 & 0.67 & -13.05 \\
\hline Ethyl acetimidate & 1.40 & 8.07 & -5.03 \\
\hline EDTA & 1.92 & 8.67 & -5.69 \\
\hline Glucosamine & 0.32 & 2.11 & -8.14 \\
\hline Triton X-100 & 0.09 & 1.05 & -14.48 \\
\hline Cysteine & 1.82 & 8.51 & -5.54 \\
\hline Tween 20 & 0.07 & 0.97 & -14.01 \\
\hline guanidinium hydrochloride & 0.38 & 2.74 & -7.64 \\
\hline Tween 80 & 0.06 & 0.89 & -13.80 \\
\hline Monoiodoacetate & 0.91 & 5.72 & -6.08 \\
\hline
\end{tabular}




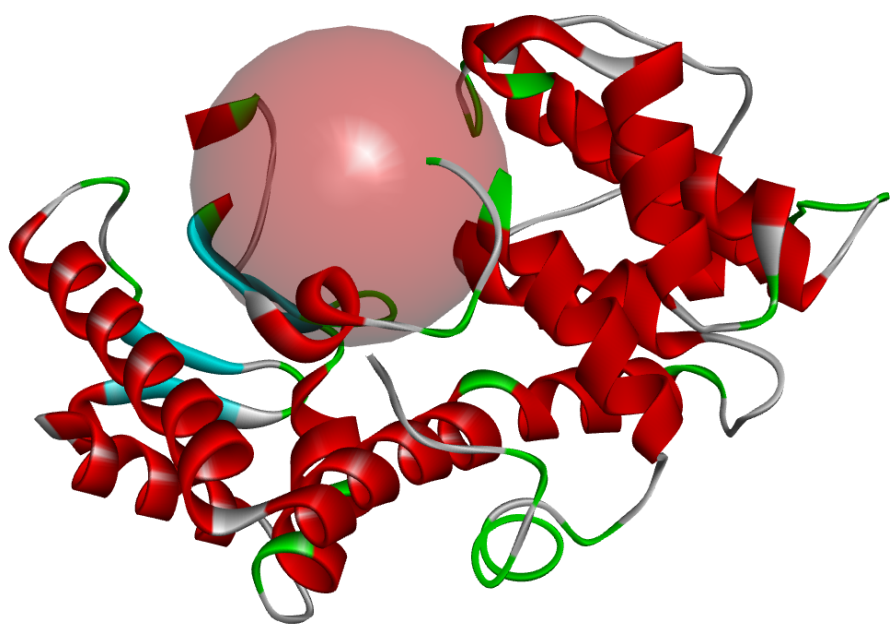

Figure 1. The docking zone. Structure of EDG1 was shown in cartoon and the docking zone was shown in red ball. The figure was generated by Discovery Studio Client 4.2.

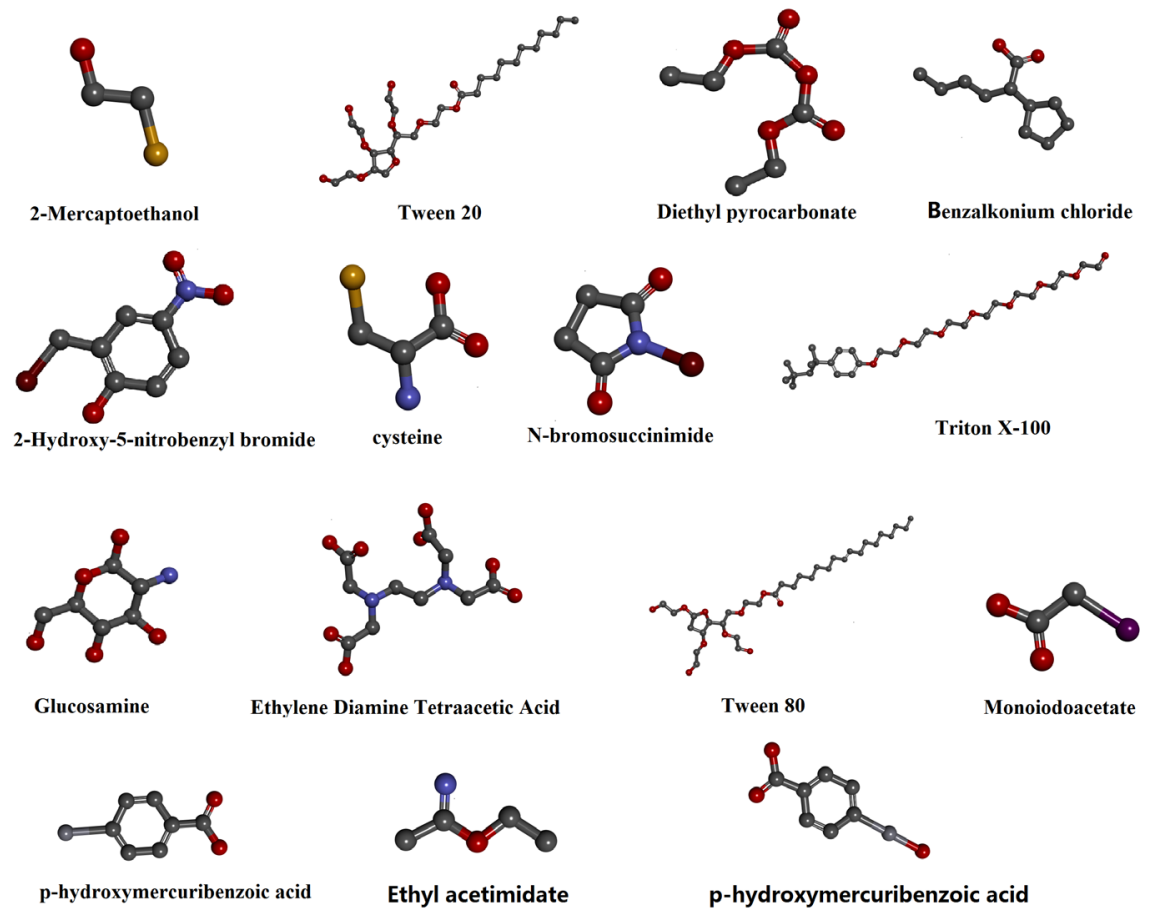

Figure 2. Chemical structures of inhibitors for Chitosanases in training set.

P-hydroxymercuribenzoic acid, 2-Mercaptoethanol, Diethyl pyrocarbonate, N-bromosuccinimide, Glucosamine, Triton X-100, 2-Hydroxy-5-nitrobenzyl bromide, Tween 20, Tween 80 and guanidinium hydrochloride, they inhibiting EAG1 with the $K_{i}$ values from 0.02 to 0.48 , were not effective inhibitors. Because the complex they formed were not stable. On the contrast, Benzalkonium chloride had strong inhibition ability and it was not suitable inhibitors to improve EAG1 stability. We docked these compounds to MP by using the Autodock4.2. The docking values of $E_{i}$ and $K_{i}$ of the favorite cluster were extracted and listed in Table 1. Considering the above factors, the restrictive condition of docking $E_{i}$ 
is not less than $-6.00 \mathrm{kcal} / \mathrm{mol}$ and not greater than $-5.00 \mathrm{kcal} / \mathrm{mol}$, the docking $K_{i}$ not less than $8 \mu \mathrm{M}$ and not greater than $9 \mu \mathrm{M}$ was set as the "initial filter" for EAG1 inhibitor selection.

\section{Virtual docking screening combined with consensus scoring}

The free commercially available compounds database ZINC, of about 2,300,000 compounds, was used as the docking library. The tertiary structures of these compounds included in the docking library were downloaded strictly form the database ZINC. Those compounds with both dock energy-score and $K_{i}$ score meeting the selective conditions $\left(-6.000 \leq E_{i} \leq-5.000,8 \leq K_{i} \leq 9\right)$ were chosen as the primary selection list. After this selection, about 5000 compounds were selected by docking scores from the pool of docking library.

The X-score [16] program, which computed the binding affinities of the given ligand molecules to their target protein, was applied to reevaluate the primary selected molecules following "initial filter" screening. The calculated score value could somewhat remove the score bias caused by single docking and was considered more accurate and possible to reflect the actual binding ability. The multiple conformations of 5000 compounds were then calculated and reevaluated by Xcscore to generate the final "hit lists". All compounds with calculated score higher than 5.0 and ranked in the top 500 for docking $E_{i}$ and Xcscore results were kept in the hit lists. Finally, the top three compounds were selected (Table 2) and purchased for further assay.

\section{Chitosanases assay}

\section{Enzyme purification}

EAG1 was purified by ultrafiltration membrane and $\mathrm{Ni}^{2+}$-charged $1 \mathrm{ml} \mathrm{His}$ Trap FF crude column (GE Healthcare), According to the methods from Sheng et al. [7] Chitosanase activity was determined at $40^{\circ} \mathrm{C}$ by estimating the amount of the reducing ends of sugars using a modified dinitrosalicyclic acid (DNS) method with glucosamine $\cdot \mathrm{HCl}$ as the calibration standard. It was measured in $0.2 \mathrm{~m} \mathrm{~L}$ of reaction mixture that contained $100 \mathrm{mM}$ acetate buffer, $0.2 \%$ chitosan, and suitably diluted enzyme. One unit of chitosanase was defined as the amount of enzyme required to liberate $1 \mu \mathrm{mol}$ reducing sugar per min under the conditions described above. Three replicates were performed per analysis.

The effect of Inhibitors on EAG1 activity was studied at $40^{\circ} \mathrm{C}$ using chitosan as substrate. The enzyme was pre-incubated with the inhibitors for $5 \mathrm{~min}$. The measurements were performed in $200 \mu \mathrm{L}$ final volume, at $40^{\circ} \mathrm{C}$ in $100 \mathrm{mM}$ acetate

Table 2. Docking scores, $\mathrm{X}$-scores and experimental $K_{i}$ values for candidates.

\begin{tabular}{ccccc}
\hline Compounds & Docking energy $(\mathrm{kcal} / \mathrm{mol})$ & Docking $K_{i}$ & Xscore & $\boldsymbol{K}_{\boldsymbol{i}}(\mu \mathrm{M})$ \\
\hline ZINC00344328 & 168.04 & -5.15 & 6.87 & \\
ZINC20476815 & 124.22 & -5.33 & 6.36 & 1.50 \\
ZINC02961282 & 101.33 & -5.45 & 6.21 & \\
\hline
\end{tabular}


buffer ( $\mathrm{pH}$ 6.0) at $50 \mathrm{ng} / \mathrm{mL}$ enzyme concentration. The Michaelis constant $(\mathrm{Km})$ for PPO was determined by Lineweaver-Burk [17] plots and Ki value was obtained from Dixon plot [8].

\section{Inhibitors}

The effect of Inhibitors on MP activity was studied at $30^{\circ} \mathrm{C}$ using Succ. Ala-Ala-Pro-Arg-AMC as substrate. The enzyme was pre-incubated with the inhibitors for $5 \mathrm{~min}$. The measurements were performed in $200 \mu \mathrm{L}$ final volume, at $30^{\circ} \mathrm{C}$ in an enzyme assay buffer (50 mM Tris- $\mathrm{HCl}(\mathrm{pH} 8.0), 10 \mathrm{mM} \mathrm{CaCl}, 0.1 \mathrm{M}$ $\mathrm{NaCl})$ at $5-30 \mathrm{ng} / \mathrm{mL}$ enzyme concentration. The activities on fluorometric substrates were measured at $380 \mathrm{~nm}$ excitation and $460 \mathrm{~nm}$ emission wave lengths [18]. The Michaelis constant $\left(K_{m}\right)$ for MP was determined by Lineweaver-Burk plots and $K_{i}$ value was obtained from Dixon plot. Boric acid and its derivations induced competitive inhibition. To describe the competitive inhibition mechanism, the Lineweaver-Burk equation in double reciprocal form can be written as:

$$
\frac{1}{v_{i}}=\frac{K_{m}}{V_{\max }}\left(1+\frac{[I]}{K_{i}}\right) \frac{1}{[S]}+\frac{1}{V_{\max }}
$$

Secondary plots can be constructed from

$$
K_{m}^{a p p}=\frac{K_{m}[I]}{K_{i}}+K_{m}
$$

Then, the $K_{i}, K_{m}$, and $V_{\max }$ values can be derived from the above equations. The secondary replots of the apparent $K_{m}$ vs. [I] is linearly fitted, assuming a single inhibition site or a single class of inhibition site [19].

\section{Results and Discussion}

The compounds isolated from the virtual screening were tested for their inhibition effects on EAG1. The top three Xscore compounds (Table 2) from the hit list were selected and ordered (or synthesized). And then, their binding affinities were measured by the assay method as mentioned above. In our experiments, the inhibition affinities for the three compounds were tested. Among them, one selected compounds turned out to inhibit the activity of EAG1 efficiently. The $K_{i}$ values for the compound were experimentally determined to $1.50 \mu \mathrm{M}$. It was reversible inhibitors of EAG1.

Under the conditions employed in the present study, the hydrolysis follows Michaelis-Menten kinetics. The kinetic parameters for the enzyme have been obtained from Lineweaver-Burk plot (Figure 3, curve- $\bullet$ ), and the results show that the $K_{m}$ and $V_{m}$ are respectively $4.97 \mu \mathrm{M}$ and $3.5772 \mu \mathrm{M} / \mathrm{min}$.

The kinetics of the enzyme in the presence of inhibitor was studied using double-reciprocal Lineweaver-Burk plots. The results (Figure 3) revealed that the value of $V_{m}$ remained the same and the value of $K_{m}$ increased with increasing inhibitor concentrations, indicating that the selected compounds induced competitive inhibition. Using Equations (1) and (2), the $K_{i}$ was calculated. 


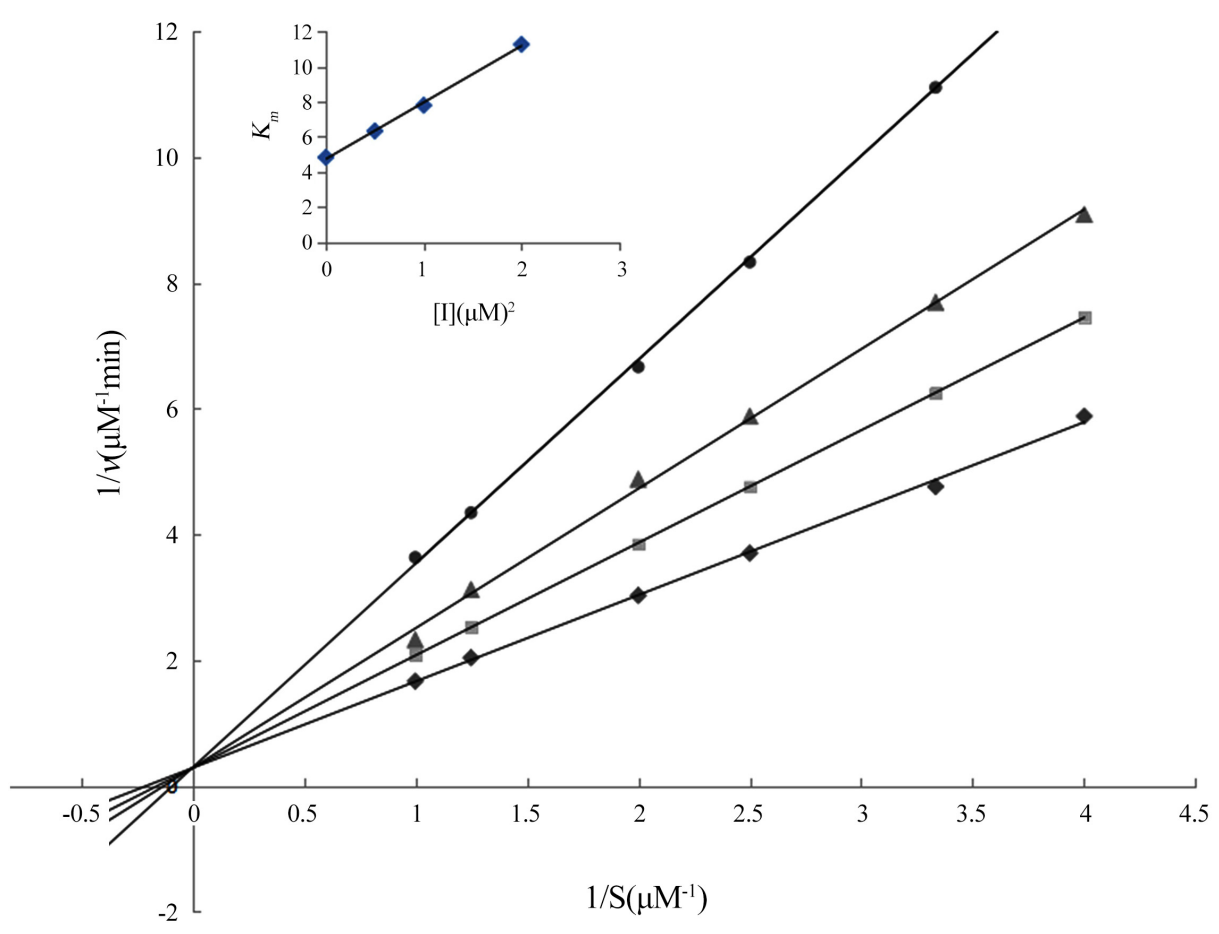

Figure 3. Plot of $1 / v$ vs. $1 /[\mathrm{S}]$. The concentrations of ZINC20476815 were 0 (- $\bullet$ ), 0.50 (---), $1.00(-\mathbf{\Delta}-)$ and $2.00(-\bullet-) \mu \mathrm{M}$, respectively. The label in figure is absolute error. The inset is the plot of $K_{m}$ vs. [I] to ascertain $K_{i}$.

\section{Conclusion}

Three potential compounds were selected and purchased for biological testing following the procedure of virtual screening and post-dock scoring. One of them was found to inhibit EAG1 in the millimole range. These active compounds served as the basis for developing inhibitors for EAG1. Overall, our results give another evidence that the effectiveness of the automated AutoDock program with the improved scoring function as a docking program for the de novo discovery of inhibitors. The method for finding EAG1's inhibitor in this study may further guide the development of inhibitors for EAG1.

\section{Acknowledgements}

This work is supported by the Zaozhuang transformation project of self-innovation and high-tech achievements (2019GH01).

\section{Conflicts of Interest}

The authors declare no conflicts of interest regarding the publication of this paper.

\section{References}

[1] Vincent, L., Hemalatha, G.R., Elodie, D., Coutinho, P.M. and Bernard, H. (2014) The Carbohydrate-Active Enzymes Database (CAZy) in 2013. Nucleic Acids Research, 42, D490-D495. https://doi.org/10.1093/nar/gkt1178 
[2] Joseph, S.R. (1996) Chitosanases-Properties and Applications: A Review. Bioresource Technology, 55, 35-45.

[3] Svendsen, A. and Clausen, I.G. (1997) Detergent Compositions Containing Protease and Novel Inhibitors for Use There.US Patent 5674833.

[4] Schulz, P., Schwadtke, K. and Smulders, E. (1990) Process for the Preparation of a Storage-Stable Liquid Detergent Composition. US Patents US4929380A.

[5] Stoner, M.R., Dale, D.A., Gualfetti, P.J., Becker, T., Manning, M.C., Carpenter, J.F., et al. (2004) Protease Autolysis in Heavy-Duty Liquid Detergent Formulations: Effects of Thermodynamic Stabilizers and Protease Inhibitors. Enzyme and Microbial Technology, 34, 114-125. https://doi.org/10.1016/j.enzmictec.2003.09.008

[6] Ji, X., Zheng, Y., Wang, W., Sheng, J., Hao, J. and Sun, M. (2013) Virtual Screening of Novel Reversible Inhibitors for Marine Alkaline Protease MP. Journal of Molecular Graphics and Modelling, 46, 125-131. https://doi.org/10.1016/j.jmgm.2013.10.004

[7] Sheng, J., Ji, X., Zheng, Y., Wang, Z. and Sun, M. (2016) Improvement in the Thermostability of Chitosanase from Bacillus ehimensis by Introducing Artificial Disulfide Bonds. Biotechnology Letters, 38, 1809-1815.

https://doi.org/10.1007/s10529-016-2168-2

[8] Dixon, M. (1953) The Determination of Enzyme Inhibitor Constants. Biochemical Journal, 55, 170-171. https://doi.org/10.1021/ci049714+

[9] Irwin, J.J. and Shoichet, B.K. (2005) ZINC-A Free Database of Commercially Available Compounds for Virtual Screening. Journal of Chemical Information and Modeling, 45, 177-182. https://doi.org/10.1021/ci049714+

[10] Irwin, J.J., Sterling, T., Mysinger, M.M., Bolstad, E.S. and Coleman, R.G. (2012) ZINC: A Free Tool to Discover Chemistry for Biology. Journal of Chemical Information and Modeling, 52, 1757-1768. https://doi.org/10.1021/ci3001277

[11] Hu, X. and Shelver, W.H. (2003) Docking Studies of Matrix Metalloproteinase Inhibitors: Zinc Parameter Optimization to Improve the Binding Free Energy Prediction. Journal of Molecular Graphics and Modelling, 22, 115-126. https://doi.org/10.1016/S1093-3263(03)00153-0

[12] Hu, X., Balaz, S. and Shelver, W.H. (2004) A Practical Approach to Docking of Zinc Metalloproteinase Inhibitors. Journal of Molecular Graphics and Modelling, 22, 293-307. https://doi.org/10.1016/j.jmgm.2003.11.002

[13] Morris, G.M., Huey, R., Lindstrom, W., Sanner, M.F., Belew, R.K., Goodsell, D.S., et al. (2009) AutoDock4 and AutoDockTools4: Automated Docking with Selective Receptor Flexibility. Journal of Computational Chemistry, 30, 2785-2791. https://doi.org/10.1002/jcc.21256

[14] Word, J.M., Lovell, S.C., Richardson, J.S. and Richardson, D.C. (1999) Asparagine and Glutamine: Using Hydrogen Atom Contacts in the Choice of Side-Chain Amide Orientation. Journal of Molecular Biology, 285, 1735-1747.

https://doi.org/10.1006/jmbi.1998.2401

[15] Pence, H.E. and Williams, A. (2010) ChemSpider: an Online Chemical Information Resource. Journal of Chemical Education, 87, 1123-1124. https://doi.org/10.1021/ed100697w

[16] Wang, R., Lu, Y. and Wang, S. (2003) Comparative Evaluation of 11 Scoring Functions for Molecular Docking. Journal of Medicinal Chemistry, 46, 2287-2303. https://doi.org/10.1021/jm0203783

[17] Lineweaver, H. and Burk, D. (1934) The Determination of Enzyme Dissociation 
Constants. Journal of the American Chemical Society, 56, 658-666. https://doi.org/10.1021/ja01318a036

[18] Massaoud, M.K., Marokházi, J. and Venekei, I. (2011) Enzymatic Characterization of a Serralysin-Like Metalloprotease from the Entomopathogen Bacterium, Xenorhabdus. Biochimica et Biophysica Acta (BBA)-Proteins and Proteomics, 1814, 1333-1339. https://doi.org/10.1016/j.bbapap.2011.05.008

[19] Si, Y., Wang, Z., Park, D., Chung, H.Y., Wang, S., Yan, L., et al. (2012) Effect of Hespaeretin on Tyrosinase: Inhibition Kinetics Integrated Computational Simulation Study. International Journal of Biological Macromolecules, 50, 257-262.

https://doi.org/10.1016/j.ijbiomac.2011.11.001 